\title{
Efficacy of daytime continuous positive airway pressure titration in severe obstructive sleep apnoea
}

\author{
J.C. Rudkowski, P. Verschelden, R.J. Kimoff
}

\begin{abstract}
Efficacy of daytime continuous positive airway pressure titration in severe obstructive sleep apnoea. J. C. Rudkowski, P. Verschelden, R. J. Kimoff. (C) ERS Journals Ltd 2001. ABSTRACT: The aim of this study was to evaluate manual nasal continuous positive airway pressure (nCPAP) titration during daytime polysomnography compared with conventional overnight titration for patients with severe obstructive sleep apnoea.

Thirty-two patients who underwent daytime titration were retrospectively matched (for age, sex, body mass index and apnoea/hypopnoea index (AHI)) to a group titrated overnight during the same period. Successful titration was defined as the identification of the nCPAP level (effective $\mathrm{nCPAP}(P$ eff $)$ ) required to eliminate respiratory events during all sleep stages. After 3 months of therapy on nCPAP at Peff, nCPAP utilization history was obtained and a group of patients underwent a repeat polysomnogram (PSG) and completed a follow-up Epworth Sleepiness Scale (ESS) score.

Initial titration was successful in $91 \%$ of daytime patients and $91 \%$ of overnight patients. The success of daytime titration was not related to diagnostic AHI or ESS score. Subjective nCPAP utilization was statistically similar in both groups. On the follow-up PSG, there were no significant differences between daytime $(n=11)$ and overnight $(n=11)$ patients in measures of sleep quality or respiratory disturbance. Both groups demonstrated similar and significant improvements in ESS score.

These findings suggest that the effective nasal continuous positive airway pressure can be accurately established during daytime titration in a substantial proportion of severe, symptomatic obstructive sleep apnoea patients.

Eur Respir J 2001; 18: 535-541.
\end{abstract}

Respiratory Division, McGill University Health Centre, Royal Victoria Hospital, Montréal, Quebéc, Canada.

Correspondence: R.J. Kimoff, Respiratory Division, Room L4.08, MUHCRoyal Victoria Hospital, 687 Pine Ave West, Montreal, Quebec, Canada, H3A $1 \mathrm{~A} 1$.

Fax: 5148431695

Keywords: Polysomnography positive-pressure respiration sleep apnoea syndromes

Received: July 202000

Accepted after revision May 12001

This study was supported by Vitalaire Montreal and ResMed Inc.
The prevalence of obstructive sleep apnoea (OSA) in the general population has been estimated at $2 \%$ of females and $4 \%$ of males [1]. There is a growing awareness of OSA on the part of both the general public and medical practitioners, which has in turn led to increasing demands upon sleep laboratories and lengthening waiting lists for diagnostic and therapeutic sleep studies. This can be especially problematic in cases where urgent diagnosis and initiation of treatment of OSA are indicated at the time of clinical evaluation (i.e. strong history for OSA with incapacitating daytime sleepiness or active cardiovascular disease).

The current treatment of choice for OSA is nasal continuous positive airway pressure (nCPAP). Conventional practice in many laboratories has been to perform an attended, full-night, manual nCPAP titration polysomnogram (PSG) prior to the initiation of home treatment. While this approach offers many advantages, it is time-consuming and expensive. A variety of alternative approaches have been described including: split-night diagnostic/titration studies [2-6], laboratory titration using automated nCPAP devices [7-10], home attended manual or unattended automated titration [11-13], and empiric treatment using prediction formulae [14]. Studies to date have indicated that there are potential advantages, but also limitations, to each of these approaches; and considerable work is required to establish the shortand long-term therapeutic efficacy of these alternate methods.

Another approach that has received relatively little attention is to conduct laboratory nCPAP titration during the daytime in patients with OSA and marked excessive daytime sleepiness. This has the potential advantage of occupying sleep laboratory facilities at a time when they may be under-utilized, thus enhancing access to treatment, and at the same time providing the potential advantages of attended adjustment of nCPAP.

Several previous reports have evaluated the reliability of diagnostic PSGs performed during the daytime [15-17]. SÉRIÈs et al. [15] demonstrated that daytime PSGs showed a high specificity for the detection of OSA and reliably estimated severity in terms of the number of events, while tending to underestimate the severity of oxygen desaturation compared with nocturnal PSGs.

Several years ago, because of the heavy demand upon the authors' sleep laboratory and long waiting lists for overnight studies, daytime nCPAP titrations were performed in patients with proven severe OSA with moderate-to-marked excessive daytime sleepiness. Many patients requiring urgent diagnosis were undergoing daytime diagnostic PSGs. Split-night studies were being used as well, but there remained 
symptomatic patients, for whom an overnight slot was not available without considerable delay. The initial impression was that daytime nCPAP titrations were relatively successful in terms of establishing an effective nCPAP level in patients with severe OSA. The intent of the present study was to formally evaluate the success of this approach.

During the time the present study was being performed, another report on daytime titration appeared [18]. These investigators described 14 OSA patients titrated in the daytime and matched to a group of similar patients titrated overnight. They found comparable sleep architecture, reduction in sleep-disordered breathing, and similar therapeutic pressures during the nCPAP titration. The two groups also demonstrated similar compliance with nCPAP therapy following 1 week of treatment. RosENTHAL et al. [18] did not, however, evaluate the long-term efficacy of the prescribed nCPAP pressure or the long-term effect on symptoms of excessive daytime sleepiness.

The specific aims of the present study were: 1) to evaluate the success of manual daytime nCPAP titration in comparison with conventional overnight titration in a large group of patients with severe OSA and complaints of excessive daytime sleepiness, and 2) to determine whether the efficacy of nCPAP titration is similar to that of patients titrated overnight as demonstrated on a follow-up overnight PSG, subjective nCPAP utilization and improvement in reported sleepiness.

\section{Methods}

\section{Subjects}

Patients were identified from a sleep disorders clinic and laboratory based in the respiratory division of a tertiary care centre. The clinic was staffed by three pulmonologists and one psychiatrist trained in sleep disorders. Fifty-eight patients were scheduled for daytime nCPAP titration at their sleep physician's discretion over a 2-yr period from January 1996December 1998. The decision to refer patients for daytime nCPAP titration was based on clinical symptoms suggestive of severe OSA with complaints of marked excessive daytime sleepiness. One patient requested daytime titration due to working overnight shifts on a regular basis. Individual sleep physicians utilized the daytime titration option to different extents. Therefore, it was also possible to retrospectively select an overnight titration comparison group from similar patients who underwent manual overnight nCPAP titration during the same time period. The selection of overnight cases was based solely on data obtained at the time of the diagnostic study before the outcome of the nCPAP titration was known. Patients were matched for sex, age ( $\pm 10 \mathrm{yrs})$, body mass index $(\mathrm{BMI})\left( \pm 5 \mathrm{~kg} \cdot \mathrm{m}^{-2}\right)$, and severity (mild: apnoea/hypopnoea index (AHI) of 15-30 events $\cdot h^{-1}$, moderate: AHI of $31-60$ events $\cdot h^{-1}$, or severe: AHI of $>60$ events $\cdot h^{-1}$ ). Suitable matches were obtained from the overnight titration group for 32 of
58 daytime titration patients. The daytime and overnight groups of 32 patients each, are referred to as the "matched" groups. The entire group of daytime patients studied $(n=58)$ are referred to as the "total" daytime group.

Patients were excluded from participating in the study if they had an AHI of $<15$ events $\cdot h^{-1}$, had any prior treatment for OSA, had OSA diagnosed by home oximetry, had periodic leg movements unrelated to respiratory events, or had hyperventilation requiring additional treatment (oxygen therapy or bilevel positive airway pressure ventilation). The study was approved by the Human Ethics Committee of the Royal Victoria Hospital and written consent for data utilization and agreement to consider participation in follow-up studies was obtained from all patients.

\section{Diagnostic studies}

Diagnostic data were obtained from the clinic charts. All study patients had OSA documented by diagnostic PSG (daytime or overnight). All night-time patients were diagnosed by overnight PSG $(n=32)$. Of the matched daytime group, 17 patients had daytime diagnostic PSG and 15 had overnight PSG. At the time of the diagnostic study or initial clinic visit, age, BMI, and Epworth Sleepiness Scale (ESS) score were obtained for each patient. Daytime PSG patients were not sleep-deprived and their studies commenced 09:00-10:00 h. Standard PSG was performed using electroencephalogram (EEG) leads C4-A1/C3-A2, right and left electroculogram (EOG), chin and anterior tibialis electromyograms (EMGs), nasal cannula pressure (Validyne P300D, Roxon, Montreal, Quebec, Canada) [19], thoracoabdominal movements by inductive plethysmography (Respitrace Systems, Respitrace Corp., Ardsley, USA) or piezoband (EPM Systems, Midlothian, VA, USA), body position by mercury switch (EPM Systems) and video monitoring, snoring via a microphone placed above the patient's head, and arterial oxyhaemoglobin saturation by finger pulse oximetry (Ohmeda Biox 3700, Ohmeda Corp., Boulder, CO, USA). All signals were acquired using a computerized system (Sandman, Melville/ Nellcor Puritan Bennett, Ottawa, Ontario, Canada) and scored manually by trained, experienced technologists with physician review. Sleep stages were identified according to standard criteria [20]. Snoring was graded as present or absent during each $30-\mathrm{s}$ epoch. Apnoeas were scored as episodes of $>90 \%$ reduction in airflow lasting $\geq 10 \mathrm{~s}$. Apnoeas were considered obstructive in the presence of persistent respiratory effort and central in the absence of effort. Hypopnoeas were defined as a period of $>10 \mathrm{~s}$ of reduction in airflow and/or inspiratory flattening of the airflow signal, with either $>2 \%$ desaturation or arousal.

Determination of the effective nasal continuous positive airway pressure

Manual nCPAP titration was performed according to American Thoracic Society Guidelines [21]. The 
sleep technician instructed the patient in the use and application of the nCPAP mask. In the laboratory, patients were requested to maintain the supine position as much as possible during sleep. The same recording configuration was used as described for the diagnostic PSG, except that mask pressure was substituted for nasal cannula pressure. Once the patient was asleep and apnoeas confirmed, the nCPAP level was started at $5 \mathrm{cmH}_{2} \mathrm{O}$ and gradually increased to eliminate apnoeas, hypopnoeas, snoring, ribcage paradox, and respiratory-related arousals (i.e. flowlimited events) in rapid eye movement (REM) and non-REM sleep, in all positions. If mask leak occurred, the technician corrected it and mouth leak was corrected by the application of a chinstrap. The maximum study duration was $8 \mathrm{~h}$ for the overnight patients and $4 \mathrm{~h}$ for the daytime patients.

After completion of the titration study, patients were prescribed nCPAP at the effective nCPAP (P eff) determined by the treating sleep physician from the PSG titration record. The Peff was defined as the pressure that alleviated apnoeas, hypopnoeas, and respiratory-related arousals (i.e. flow-limited events) during non-REM and REM sleep. Adequate sleep architecture was defined as total sleep time (TST) $>90 \mathrm{~min}$ and REM sleep time $>10 \mathrm{~min}$. With respect to body position, all patients were documented to be supine during $P$ eff on the titration study. Studies were deemed "successful" if the $P$ eff could be confidently determined (i.e. a pressure could be identified, which clearly alleviated upper airway obstructive events as described above) and the sleep architecture was adequate. Studies were deemed "partially successful" if the patient had inadequate REM sleep (i.e. $<10 \mathrm{~min}$ ), but events were eliminated during the sleep that was recorded. If the patient did not achieve adequate REM sleep during the nCPAP titration PSG, and REM-dominant OSA was demonstrated on the diagnostic PSG, additional pressure $\left(1-2 \mathrm{cmH}_{2} \mathrm{O}\right)$ was added to the $P$ eff for the nCPAP prescription. This approach was based on data on non-REM versus REM pressure requirements from the authors' laboratory (unpublished observations), which is consistent with that of other centres $[22,23]$. If the patient did not achieve adequate REM sleep in either the diagnostic or nCPAP titration PSG, the $P$ eff was determined from the non-REM sleep recorded during the titration study and no additional pressure was added to $P$ eff. Unsuccessful studies were classified in the following manner: 1) complete inability to sleep (i.e. because of inability to tolerate the nCPAP mask), 2) technical failure (i.e. computer error), or 3) true failure (i.e. patient slept adequately, but nCPAP was unable to alleviate the respiratory disturbances).

\section{Home treatment and follow-up}

After receiving a prescription for $\mathrm{nCPAP}$ at $P$ eff, patients were referred to local nCPAP providers for initiation of home treatment. After $>12$ weeks of home nCPAP use, patients were contacted by phone and asked to return for an overnight PSG on their own machine at the prescribed Peff. Patients who agreed to return underwent an overnight PSG on their own nCPAP machine with the same recording configuration as described above. The nCPAP level was not adjusted during the follow-up study, even if events were observed. The treating physician could modify the nCPAP pressure at their discretion after the follow-up study. At this time, the BMI and ESS score were also remeasured. All patients were contacted regarding subjective nCPAP utilization. At the time this study was undertaken, the majority of patients did not have objective monitoring devices available in their nCPAP machines. A patient was deemed to be a regular user of nCPAP if: 1) the machine had been installed in his/her home, 2) they stated that it was worn $>5$ nights per week, and 3) the patient had been using nCPAP for $>3$ months.

\section{Data analysis}

The matched groups of overnight and daytime patients were compared in terms of anthropomorphic indices, reported symptoms of excessive daytime sleepiness (ESS score), AHI, arousal index (AI), and nadir saturation at baseline. The nCPAP titrations and follow-up PSGs of each group were compared in terms of sleep duration, sleep architecture, sleep quality, and respiratory disturbances. Group data are shown as mean \pm SEM. All parametric data were compared using the paired t-test unless otherwise stated. Changes in the ESS score at follow-up were compared by Chi-squared analysis. Comparison of the success of titration and rates of utilization of nCPAP for the two groups was made using the MannWhitney rank sum test. Differences were considered significant with a p-value of $<0.05$.

\section{Results}

The baseline characteristics of the matched overnight and daytime titration groups are shown in table 1 . There were six females and 26 males in each group. There were no statistically significant differences between the groups. During the nCPAP titration, the daytime group had a TST of $2.1 \pm 0.1 \mathrm{~h}$ versus $4.8 \pm 03 \mathrm{~h}$ for the overnight group $(\mathrm{p}<0.001)$ (table 2$)$.

Table 1.-Baseline characteristics (at time of diagnostic study)

\begin{tabular}{lccc}
\hline & Day & Night & p-value \\
\hline Age yrs & $49.9 \pm 1.9$ & $49.9 \pm 2.0$ & NS \\
BMI kg $\cdot \mathrm{m}^{-2}$ & $34.7 \pm 1.4$ & $32.9 \pm 1.5$ & NS \\
ESS & $14.4 \pm 1.4$ & $12.2 \pm 1.0$ & NS \\
AHI events $\cdot \mathrm{h}^{-1}$ & $76.4 \pm 6.2$ & $61.6 \pm 4.8$ & NS \\
AI arousals $\cdot \mathrm{h}^{-1}$ & $66.0 \pm 5.9$ & $54.5 \pm 5.0$ & NS \\
Nadir $\mathrm{S}_{\mathrm{a}, \mathrm{O}_{2}} \%$ & $75.6 \pm 2.2$ & $81.3 \pm 1.9$ & NS \\
\hline
\end{tabular}

Data are presented as mean \pm SEM. BMI: body mass index; ESS: Epworth Sleepiness Scale (maximum score=24); AHI: apnoea/hypopnoea index; AI: arousal index; $S_{\mathrm{a}} \mathrm{O}_{2}$ : arterial oxygen saturation; NS: nonsignificant. Data were compared using the t-test. 
Table 2. - Nasal continuous positive airway pressure (nCPAP) titration study characteristics

\begin{tabular}{|c|c|c|c|}
\hline & Day & Night & p-value \\
\hline AHI events $\cdot h^{-1}$ & $29.4 \pm 4.1$ & $20.3 \pm 3.3$ & NS \\
\hline AI arousals $\cdot h^{-1}$ & $33.9 \pm 4.5$ & $27.4 \pm 3.9$ & NS \\
\hline TST h & $2.1 \pm 0.1$ & $4.8 \pm 0.3$ & $<0.001$ \\
\hline AHI $P$ eff events $\cdot h^{-1}$ & $18.2 \pm 4.7$ & $11.7 \pm 2.5$ & NS \\
\hline AI $P$ eff arousals $\cdot h^{-1}$ & $19.7 \pm 5.5$ & $15.3 \pm 3.0$ & NS \\
\hline Sleep at $P$ eff $\mathrm{h}$ & $1.0 \pm 0.5$ & $2.5 \pm 2.1$ & $<0.001$ \\
\hline Sleep effciency $\%$ & $75.8 \pm 3.0$ & $65.2 \pm 3.4$ & $<0.05$ \\
\hline Stage $1 \%$ & $15.9 \pm 2.3$ & $13.1 \pm 2.1$ & NS \\
\hline Stage $2 \%$ & $44.7 \pm 2.8$ & $54.7 \pm 2.7$ & $<0.01$ \\
\hline Stages 3 and $4 \%$ & $21.1 \pm 2.8$ & $17.0 \pm 2.1$ & NS \\
\hline REM \% & $18.2 \pm 2.8$ & $17.9 \pm 1.5$ & NS \\
\hline Nadir $\mathrm{Sa}_{\mathrm{a}, \mathrm{O}_{2}} \%$ & $88.5 \pm 0.5$ & $85.8 \pm 1.3$ & NS \\
\hline$P$ eff $\mathrm{cmH}_{2} \mathrm{O}$ & $9.7 \pm 0.6$ & $9.2 \pm 0.5$ & NS \\
\hline
\end{tabular}

Data are presented as mean \pm SEM. AHI: apnoea/hypopnoea index; AI: arousal index; TST: total sleep time; REM: rapid eye movement; $P$ eff: effective nCPAP; $S_{\mathrm{a}, \mathrm{O}_{2}}$ : arterial oxygen saturation; NS: nonsignificant. Data were compared using the t-test.

The $P$ eff for each group was similar. Both groups slept at $P$ eff for $\sim 50 \%$ of the total sleep time. The AHI and AI during sleep at $P$ eff were not significantly different between the daytime and overnight titration groups.

The matched daytime group $(n=32)$ was representative of the total group of daytime patients studied $(n=58)$. The characteristics at the time of diagnosis for the total daytime group were as follows: average age, $51.0 \pm 1.7 \%$ yrs; BMI, 37.7 $\pm 1.4 \mathrm{~kg} \cdot \mathrm{m}^{-2}$; ESS score, 15.4 \pm 0.9 ; AHI, 84.0 \pm 5.7 events $\cdot \mathrm{h}^{-1}$, and nadir saturation, $76.5 \pm 1.6 \%$. None of these values were significantly different from those of the matched daytime subjects (table 1). The nCPAP titration study characteristics were also similar for the total group: TST, $1.98 \pm 0.1$ h; REM, $17.3 \pm 1.9 \%$; AHI, $30.1 \pm 3.3$ events $\cdot h^{-1}$; nadir saturation, $86.8 \pm 1.6 \%$; and $P$ eff, $10.2 \pm 0.5 \mathrm{cmH}_{2} \mathrm{O}$. There were no statistically significant differences between the values obtained for the total daytime group and the matched daytime group (table 2).

Success of the nCPAP titration was judged by the criteria outlined in the previous section. Using the strictly defined criteria, a slightly higher percentage of patients were titrated successfully overnight than during the daytime, but this was not statistically significant $(p=0.70$, table 3 ). The proportion of successful titrations was equivalent if patients classified as having "partially successful" titrations were included. These partially successful patients did not achieve adequate REM sleep (i.e. $>10 \mathrm{~min}$ ) during the nCPAP titration PSG, so that the $P$ eff was determined from the period of non-REM sleep that was recorded, as described previously. The majority of the unsuccessful patients for whom a $P$ eff could not be determined, failed to achieve sleep due to an inability to tolerate the nCPAP mask (table 3 ). This occurred in three patients in each group. There was one technical failure in each group secondary to computer malfunction and in no patient was failure due to the inability to alleviate obstructive events with nCPAP. The success of the total daytime group (unmatched
Table 3. - Success of day and night titrations

\begin{tabular}{lcc}
\hline & Day & Night \\
\hline Total n & 32 & 32 \\
Succcessful & $22(69)$ & $27(84)$ \\
Partial success & $6(19)$ & $1(3)$ \\
Combined success & $28(91)$ & $28(91)$ \\
Unsuccessful & $4(13)$ & $4(13)$ \\
Did not sleep & $3(9)$ & $3(9)$ \\
Technical failure & $1(3)$ & $1(3)$ \\
True failure & $0(0)$ & $0(0)$ \\
\hline
\end{tabular}

Data are presented as $n(\%)$. There were no significant differences between the two groups with the Mann-Whitney rank sum test $(\mathrm{p}=0.70)$.

plus matched patients) was $82 \%$ compared to $91 \%$ $(p=0.55)$ for the matched group of day subjects.

In terms of subjective nCPAP utilization, 21 of 32 $(66 \%)$ daytime patients and 19 of $32(59 \%)$ overnight patients stated that they were using nCPAP regularly $>3$ months after initiating therapy (table 4). Nine of $32(28 \%)$ daytime and 12 of $32(38 \%)$ overnight patients were not on nCPAP. These differences were not statistically significant $(\mathrm{p}=0.84)$. Most of these patients had refused therapy altogether and a minority had chosen alternate therapies or discontinued nCPAP after a trial of therapy. Few patients were lost to follow-up in either group. When nCPAP utilization was assessed, specifically in patients who underwent a successful nCPAP titration, comparable proportions of patients were using nCPAP in each group (data not shown).

Follow-up overnight PSG studies were obtained for 11 daytime and 11 overnight patients. For both the daytime and overnight groups, patients who returned for follow-up were compared with those who did not in terms of baseline characteristics (those variables shown in table 1) and the response to initial nCPAP titration (those variables shown in table 2), and no significant differences were found. Thus, the follow-up patients were representative of the overall matched groups for both the daytime and overnight subject. The results from the follow-up studies are shown in table 5. Measures of respiratory disturbance, sleep quality, and sleep architecture were not significantly different between the daytime and overnight

Table 4.-Nasal continuous positive airway pressure (nCPAP) utilization

\begin{tabular}{lcc}
\hline & Day & Night \\
\hline Patients n & 32 & 32 \\
On nCPAP & $21(66)$ & $19(59)$ \\
Not on nCPAP & $9(28)$ & $12(38)$ \\
$\quad$ Never on & $7(22)$ & $10(31)$ \\
Other treatment & $1(3)$ & $0(0)$ \\
Discontinued & $1(3)$ & $2(6)$ \\
Lost to follow-up & $2(6)$ & $1(3)$ \\
\hline
\end{tabular}

Data are presented as $\mathrm{n}(\%)$ unless otherwise stated. There were no significant differences between the two groups with the Mann-Whitney rank sum test $(\mathrm{p}=0.84)$. 
Table 5. - Follow-up studies

\begin{tabular}{lccc}
\hline & Day & Night & p-value \\
\hline Patients n & 11 & 11 & \\
AHI events $\cdot \mathrm{h}^{-1}$ & $9.8 \pm 2.5$ & $13.9 \pm 3.7$ & NS \\
AI arousals $\cdot \mathrm{h}^{-1}$ & $19.2 \pm 5.0$ & $23.6 \pm 5.3$ & NS \\
Nadir $\mathrm{Sa}_{\mathrm{a}, \mathrm{O}_{2} \%} \%$ & $88.6 \pm 1.3$ & $90.5 \pm 1.1$ & NS \\
Sleep efficiency \% & $76.9 \pm 4.4$ & $81.5 \pm 3.1$ & NS \\
Stage changes & $99.6 \pm 11.7$ & $109.4 \pm 17.5$ & NS \\
Wakenings & $19.3 \pm 4.1$ & $21.7 \pm 5.6$ & NS \\
TST h & $4.8 \pm 0.4$ & $5.8 \pm 0.2$ & 0.05 \\
Stage $1 \%$ & $9.4 \pm 1.6$ & $10.2 \pm 3.2$ & NS \\
Stage 2\% $\%$ & $58.7 \pm 2.0$ & $60.2 \pm 2.8$ & NS \\
Stages 3 and $4 \%$ & $12.7 \pm 2.2$ & $12.0 \pm 2.1$ & NS \\
REM \% & $19.1 \pm 2.2$ & $17.6 \pm 2.2$ & NS \\
Peff $\mathrm{cmH}_{2} \mathrm{O}$ & $11.4 \pm 0.94$ & $9.5 \pm 0.8$ & NS \\
\hline
\end{tabular}

Data presented as mean \pm SEM. AHI: apnoea/hypopnoea index; AI: arousal index; $\mathrm{Sa}_{\mathrm{a}} \mathrm{O}_{2}$ : arterial oxygen saturation; TST: total sleep time; REM: rapid eye movement; Peff: effective nasal continuous positive airway pressure; NS: nonsignificant. Data were compared using the t-test.

follow-up groups, except for a slightly longer TST in the overnight group (table 5). Based on the statistical variability of the measures obtained, this number of subjects yielded a statistical power of 0.84 to identify a difference in AHI of 10 events $\cdot \mathrm{h}^{-1}$ on the follow-up PSG between the two groups.

In terms of subjective complaints of excessive daytime sleepiness, all patients improved to the same degree. The daytime titration follow-up group had an improvement in ESS scores from $18.9 \pm 1.3$ to $8.7 \pm 1.3$ $(\mathrm{p}<0.001)$ as did the overnight follow-up group (13.7 \pm 1.3 to $7.3 \pm 2.2, \mathrm{p}<0.03)$. The degree of improvement determined by the magnitude of decrease of the ESS from diagnosis to follow-up was not statistically different between the two groups at $54 \%$ and $47 \%$, respectively.

A post hoc analysis of the successful and unsuccessful daytime titrations (using both the strict criteria and the total group of successful titrations) was conducted, and revealed that the success of nCPAP titration could not be related to any of the following variables at the time of diagnosis: RDI, nadir saturation, BMI, and ESS score (table 6). There was a trend towards an increased diagnostic ESS in successful patients $(15.9 \pm 1.5$ versus $10.2 \pm 2.3)$, but this did not reach statistical significance.

Table 6. - Potential predictors of daytime titration success

\begin{tabular}{llcc}
\hline & Unsuccessful & Successful & p-value \\
\hline AHI events $\cdot \mathrm{h}^{-1}$ & $65.5 \pm 13.1$ & $81.3 \pm 6.8$ & NS \\
${\text { Nadir } S \mathrm{a}, \mathrm{O}_{2} \%} \%$ & $74.0 \pm 4.6$ & $76.2 \pm 2.5$ & NS \\
BMI $\mathrm{kg} \cdot \mathrm{m}^{-2}$ & $34.9 \pm 2.6$ & $34.6 \pm 1.8$ & NS \\
ESS score & $10.2 \pm 2.3$ & $15.9 \pm 1.5$ & 0.07 \\
\hline
\end{tabular}

Data are presented as mean \pm SEM. AHI: apnoea/hypopnoea index; $\mathrm{Sa}_{\mathrm{a}} \mathrm{O}_{2}$ : arterial oxygen saturation; BMI: body mass index; ESS: Epworth Sleepiness Scale; NS: nonsignificant. All values are from diagnostic studies. Data were compared using unpaired t-testing.

\section{Discussion}

This study demonstrates that daytime nCPAP titration is a feasible alternative to conventional overnight titration for severe, symptomatic OSA patients. The success of titration was measured qualitatively as the ability of the physician to determine the $P$ eff from the titration study. Peff could be determined in a similar proportion of subjects and the rates of subjective nCPAP utilization did not differ between these two groups after $>3$ months of nCPAP therapy. Although it would have been ideal to measure objective compliance, this was not possible because the patients did not have nCPAP machines equipped with monitoring devices, due to the retrospective design of the study. It is unclear why any bias towards overreporting of nCPAP use would be different between daytime and overnight titration groups. Since the same approach to assessing compliance was used for the two groups, it can be concluded that the patient responses do reflect similar long-term compliance for patients titrated during the daytime versus overnight.

The effectiveness of the nCPAP titration was evaluated by performing follow-up PSGs with nCPAP at $P$ eff in a subset of patients from both the daytime and overnight titration groups with the patients using their own machines. Although the authors were only able to obtain follow-up overnight PSGs on 11 daytime and 11 overnight patients, these groups adequately reflected the characteristics of the total population of study patients. It was found that nCPAP treatment was similarly effective in the two groups at follow-up, as determined by reduced respiratory events and arousals, improved sleep architecture, and lower ESS scores (including a similar degree of improvement).

By performing titrations in the daytime, patients identified as having severe OSA can be titrated and treated earlier without adding to the already substantial waiting lists for overnight titration. One potential limitation of this study is that patients were not randomized prospectively to daytime versus overnight titration groups. Patients were referred for daytime nCPAP titration on the basis of their stated ability to sleep during the day. However, not all clinic physicians referred patients for daytime studies with the same frequency. It was therefore possible to identify a group of subjects titrated in the usual overnight manner who reasonably matched the daytime group with respect to disease severity. There was only one patient in the daytime group who was a shift worker. The authors believe that the presented findings accurately reflect the success of these therapeutic approaches in similar patients. Furthermore, since this study was undertaken in a clinical setting, it is likely that the subjects would be comparable to patients in other clinical settings who are believed to require urgent treatment.

RosentHAL et al. [18] recently reported a similar trial with 14 patients in each group. They measured success quantitatively by defining titration success as the resolution of respiratory events and improvement of sleep architecture on the titration study. They also obtained compliance data and reports of subjective sleepiness at 1 week after initiation of nCPAP 
treatment. They did not evaluate success qualitatively as the ability to determine $P$ eff, nor did they evaluate long-term outcomes such as the efficacy of nCPAP treatment at $P$ eff and the reduction in excessive daytime sleepiness. The present authors have studied a considerably larger group of patients (total $n=58$ ) and have confirmed that patients undergoing daytime titration have similar reductions in sleep-disordered breathing to those undergoing overnight titration (measures of quantitative success). In addition, it has been possible to demonstrate that the daytime titration studies were similar in quality to overnight studies, by assessing the ability to determine $P$ eff in a given titration study. Moreover, measures of longterm outcome were obtained by looking at subjective nCPAP utilization, follow-up PSGs, and improvement in subjective sleepiness after $>3$ months of treatment.

It is interesting that the daytime titration group achieved similar sleep architecture to the overnight group, despite a reduced TST. This has been noted in previous diurnal diagnostic studies and is usually explained by the effects of sleep deprivation [24-26]. However, the patients in this study were not sleep deprived. There were more patients in the daytime group who slept, but without adequate duration of REM sleep. This may have been due to the maximal duration of $4 \mathrm{~h}$ sleep in the daytime group. The AHI and AI at $P$ eff were similar in both groups and showed a significant improvement from the diagnostic AHI and AI (tables 1 and 2). These patients were treated effectively (confirmed during both non-REM and REM on the follow-up PSG (table 5)) and their reported nCPAP use was at a similar rate to those who achieved adequate REM. This suggests that the approach of empirically adding $1-2 \mathrm{cmH}_{2} \mathrm{O}$ to the non-REM $P$ eff value [22, 23], if REM sleep does not occur during a daytime nCPAP titration (in those patients who have significant REM-related OSA on the diagnostic PSG), provides an adequate approximation of the nCPAP requirement, which could be confirmed in a subsequent follow-up study.

While the mean TST during the daytime titration studies was significantly shorter $(2.1 \pm 0.1 \mathrm{~h})$ than for the overnight studies $(4.8 \pm 0.3 \mathrm{~h})$, it is noteworthy that the mean daytime TST value is very similar to those reported for the nCPAP segment of split-night laboratory titration studies $[2,3,5]$. Specifically, the published figures (mean \pm SD) are $2.7 \pm 0.9$ [2], $2.2 \pm 1.1$ [3], and 2.7 \pm 1.1 [5]. Thus, the approach of daytime titration provides a sleep period during which to establish the effective nCPAP level, which is very similar to that of a split-night PSG (also with, as noted previously, a similar distribution of sleep stages to overnight titration studies). Recent favourable data on intermediate-term compliance with nCPAP following split night titration [6], and thus the adequacy of titration over a sleep period of similar duration, supports the findings of the present study. The major advantage of daytime titration would be increased access to sleep laboratory facilities in centres such as the present authors' where daytime slots are readily available and overnight slots, whether for split-night or other studies, are scarce.
It would be clinically useful to be able to predict which patients with suspected OSA and a substantial complaint of excessive sleepiness would be more likely to successfully undergo daytime titration. It was not possible to demonstrate any difference between the successful and unsuccessful groups in terms of diagnostic BMI or AHI. There was a trend towards a higher ESS score in the successful group, suggesting that patients who are excessively sleepy are more likely to benefit from daytime titration. One possibility for a future study would be to prospectively assess these and other characteristics of patients undergoing daytime nCPAP titration to determine predictors of success.

Based on the findings of this study, it can be concluded that daytime nasal continuous positive airway pressure titration is a viable alternative to conventional overnight titration for patients with severe obstructive sleep apnoea requiring urgent initiation of treatment. The daytime patients in the present study were specifically selected based upon marked sleepiness and the strong impression is that this remains an essential prerequisite for successful daytime nasal continuous positive airway pressure titration. The authors believe there is sufficient evidence to recommend that daytime nasal continuous positive airway pressure titration be added to routine clinical practice as a strategy for determining the effective continuous positive airway pressure level for similar, severely sleepy patients. Daytime continuous positive airway pressure titration may therefore represent a viable approach to reducing the waiting lists and delays in initiating therapy for patients with severe, symptomatic obstructive sleep apnoea and improving the efficiency of sleep laboratory resource utilization in centres with limited overnight availability.

\footnotetext{
Acknowledgements. The authors gratefully acknowledge the contribution to this study of the Royal Victoria Hospital Sleep Laboratory technical staff including A. Olha, N. Brienza, V. Champagne, F. Pelligrini, J. Walsh and R. Wilson, as well as the assistance of D. Bernad with data collection and management.
}

\section{References}

1. Young T, Palta M, Dempsey J, Skatrud S, Weber S, Badr S. The occurrence of sleep-disordered breathing among middle-aged adults. $N$ Engl J Med 1993; 328: $1230-1235$.

2. Fleury B, Rakotonanahary D, Tehindrazanarivelo AD, Hausser-Hauw C, Lebeau B. Long-term compliance to continuous positive airway pressure (nCPAP) set up during a split-night polysomnography. Sleep 1994; 17: 512-515.

3. Sanders MH, Kern NB, Constantino JP, et al. Adequacy of prescribing positive airway pressure therapy by mask for sleep apnea on the basis of a partial-night trial. Am Rev Respir Dis 1993; 147: 11691174.

4. Strollo PJ, Sanders MH, Constantino JP, Walsh SK, Stiller RA, Atwood CW Jr. Split-night studies for the 
diagnosis and treatment of sleep-disordered breathing. Sleep 1996; 19: Suppl. 10, S255-S259.

5. Yamashiro Y, Kryger MH. CPAP titration for sleep apnea using a split-night protocol. Chest 1995; 107: 62-66.

6. Sanders MH, Constantino JP, Strollo PJ, Studnicki K, Atwood CW. The impact of split-night polysomnography for diagnosis and positive pressure therapy titration on treatment acceptance of adherence in sleep apnea/hypopnea. Sleep 2000; 23: 17-24.

7. Becker HF. Experience with new autotitrating nasal continuous positive airway pressure machines. Monaldi Arch Chest Dis 1998; 53: 582-585.

8. Lloberes P, Ballester E, Montserrat JM, et al. Comparison of manual and automatic CPAP titration in patients with sleep apnea/hypopnea syndrome. Am J Respir Crit Care Med 1996; 154: 1755-1758.

9. Stradling JR, Barbour C, Pitson DJ, Davies RJO. Automatic nasal continuous positive airway pressure titration in the laboratory: Patient outcomes. Thorax 1997; 52: 72-75.

10. Teschler H, Farhat AA, Exner V, Konietzko N, Berthon-Jones M. AutoSet nasal CPAP titration: constancy of pressure, compliance and effectiveness at 8 month follow-up. Eur Respir J 1997; 10: 2073-2078.

11. Juhász J, Schillen J, Urbigkeit A, Ploch T, Penzel T, Peter JH. Unattended continuous positive airway pressure titration. Clinical relevance and cardiorespiratory hazards of the method. Am J Respir Crit Care Med 1996; 154: 359-365.

12. White DP, Gibb TJ. Evaluation of the Healthdyne NightWatch system to titrate CPAP in the home. Sleep 1998; 21: 198-204.

13. Waldhorn RE, Wood K. Attended home titration of nasal continuous positive airway pressure therapy for obstructive sleep apnea. Chest 1993; 104: 1707-1710.

14. Sériès F, Marc I. Efficacy of automated continuous positive airway pressure that uses an estimated required pressure in the treatment of obstructive sleep apnea syndrome. Ann Intern Med 1997; 127: 588-595.

15. Sériès F, Cormier Y, La Forge J. Validity of diurnal sleep recording in the diagnosis of sleep apnea syndrome. Am Rev Respir Dis 1991; 143: 947-949.
16. Haraldsson P-O, Carenfelt C, Knutsson E, Persson HE, Rinder J. Preliminary report: validity of symptom analysis and daytime polysomnography in diagnosis of sleep apnea. Sleep 1992; 15: 261-263.

17. Goode GB, Slyter HM. Daytime polysomnogram diagnosis of sleep disorders. J Neurol Neurosurg Psychiatry 1983; 46: 159-161.

18. Rosenthal L, Nykamp K, Guido P, et al. Daytime CPAP titration. A viable alternative for patients with severe obstructive sleep apnea. Chest 1998; 114: 10561060.

19. Montserrat JM, Farre R, Ballester E, Felez MA, Pasto M, Navajas D. Evaluation of nasal prongs for estimating nasal flow. Am J Respir Crit Care Med 1997; 155: 211-215.

20. Rectschaffen A, Kales A. A manual of standardized terminology, techniques, and scoring systems for sleep stages of human subjects. Washington, D.C., Public Health Service, US Government Printing Office, 1968.

21. American Thoracic Society. Indications and standards for use of nasal continuous positive airway pressure (CPAP) in sleep apnea syndromes. Am J Respir Crit Care Med 1994; 150: 1738-1745.

22. Oksenberg A, Silverberg DS, Arons E, Radwan H. The sleep position has a major effect on optimal nasal continuous positive airway pressure. Relationship with rapid eye movements and non-rapid eye movements sleep, body mass index, respiratory disturbance index, and age. Chest 1999; 116: 1000-1006.

23. Pevernagie DA, Shepard JW Jr. Relations between sleep stage, posture and effective nasal CPAP levels in OSA. Sleep 1992; 15: 162-167.

24. Persson HE, Svanborg E. Sleep deprivation worsens obstructive sleep apnea: comparison between diurnal and nocturnal polysomnography. Chest 1996; 109: 645-650.

25. Sériès F, Roy N, Marc I. Effects of sleep deprivation and sleep fragmentation on upper airway collapsibility in normal subjects. Am J Respir Crit Care Med 1994; 150: 481-485.

26. Stoohs RA, Dement WC. Snoring and sleep-related breathing abnormality during partial sleep deprivation (letter). N Engl J Med 1993; 328: 1279. 